\title{
An Improved Thermionic Microwave Gun and Emittance-Preserving Transport Line*
}

\author{
Michael Borland \\ Argonne National Laboratory, 9700 So. Cass Ave., Argonne, IL 60439
}

\section{Abstract}

The positron/electron linac for the Advanced Photon Source (APS) at Argonne National Laboratory can be used to accelerate electrons to $650 \mathrm{MeV}$. As part of a project to use this linac to test the quality of insertion devices, work has been done to develop a higher-brightness thermionic microwave gun of the SSRL [1] type. The new gun design has smaller emittance by a factor of 3 to 4 . The ratio of cathode field to maximum surface field is more than doubled. The new alpha-magnet-based transport line design produces negligible emittance degradation due to chromatic aberrations, in spite of the $\pm 5 \%$ momentum spread.

\section{INTRODUCTION AND MOTIVATION}

The advantages of using RF fields to accelerate electrons off of a gun cathode are increasingly well-known. At S-band, surface electric fields in excess of $300 \mathrm{MV} / \mathrm{m}$ are feasible [2], more than an order of magnitude greater than possible with DC fields. The resultant rapid acceleration of electrons leads to reduced space-charge effects in the gun. RF guns produce beams with energies of several $\mathrm{MeV}$, giving reduced space-charge effects during beam transport.

Most RF gun work involves the use of laser-pulsed photocathodes to produce relatively short beam pulses directly at the cathode. While this has a number of advantages, cost, simplicity, and reliability are not currently among them. In contrast, the thermionic gun built at the Stanford Synchrotron Radiation Laboratory (SSRL) and its associated systems are based on simpler technology, yielding reliable performance that exceeds that of simple DC-gun-based injectors in beam brightness [3].

The primary mission of the APS is to provide $\mathrm{X}$-ray beams from the $7 . \mathrm{GeV}$ positron storage ring. Many of the beamlines will use undulators, which must be carefully constructed in order to produce the desired X-ray beam properties for the user without negatively impacting the positron beam dynamics. The primary purpose of the APS RF gun is to provide a high-brightness electron beam to test insertion devices prior to installation. It will also allow characterization of novel insertion devices pricr to installation. The gun will be coupled with the $650-\mathrm{MeV}$ APS linac [4], now under construction.

A number of testing techniques are under consideration. One can determine the transport properties of a device by measuring the coordinates of a beam exiting the device as a function of the input coordinates. The beam emittance can be measured before and after the device, to ascertain whether there has been degradation. The spectral properties and angular distribution of the radiation can be used as sensitive measures of the quality

* Work supported by U.S. Department of Energy. Office of Basic Energy Sciences under Contract No. W-31-109-ENG-38. of the device. The last two techniques require a low-emittance electron beam, but not necessarily a high-current one.

The normalized RMS emittance of the electron beam in the horizontal plane is defined as $\varepsilon_{\mathrm{a}, \mathrm{x}}=\pi \sqrt{\left\langle\mathrm{x}^{2}\right\rangle\left\langle\mathrm{p}_{\mathrm{x}}^{2}\right\rangle-\left\langle\mathrm{p}_{\mathrm{x}} \mathrm{x}\right\rangle^{2}}$ where $\mathrm{p}_{\mathrm{x}}=\beta_{\mathrm{x}} \gamma$ is the normalized transverse momentum in the horizontal plane. For a sufficiently mono-energetic beam, the geometric RMS emittance is $\varepsilon_{\mathrm{x}} \approx \varepsilon_{\mathrm{n}, \mathrm{x}} / \gamma$. It is well known that a necessary condition for coherent undulator radiation is $\varepsilon_{n, x} / \gamma \leq \lambda / 4$, and similarly in the $y$ plane, where $\lambda$ is the wavelength of the radiation. At the boundary of this regime, the angular and spatial distributions of the radiation are roughly equally determined by the properties of the electron beam and of the undulator.

Using the usual expression for the wavelength of undulator radiation, one sees that coherent radiation is obtained only when $\gamma \leq \lambda_{u}\left(1+K^{2} / 2\right) /\left(8 \varepsilon_{n}\right)$, where $\lambda_{n}$ is the undulator period and $K=x_{\max }^{\prime} \gamma$. Hence, fox a given normalized emittance, one can obtain coherent radiation by reducing the beam energy. However, this is at odds with the desire to produce short wavelength radiation.

Various undulators will be used at APS, with periods from $23 \mathrm{~mm}$ to $200 \mathrm{~mm}$ and $\mathrm{K}$ values between 0.3 and 2.7 [5]. With beam energies between 50 and $650 \mathrm{MeV}$, a normalized emittance of $10 \pi \cdot m_{e} c \cdot \mu m$ gives coherent radiation in the UV to IR for these undulator parameters. This emittance was chosen as the goal for the new gun and transport line design.

\section{GUN DESIGN CRITERIA}

As with the SSRL gun, the APS gun is a side-coupled, $\pi / 2$-mode, standing-wave structure, resonant at $2856 \mathrm{MHz}$, with a normalized load impedance of about 4 . In order to reduce the beam emittance, a number of aspects of the SSRL design needed to be improved. In considering what follows, the reader may wish to refer to Figures 1 and 2, which show the new cavity phase and field profiles, respectively.

1. Nonlinear radial dependence of fields. The SSRL gun cavity was based on a design optimized for high shunt impedance, so that the gun has rather long, sharp cavity noses. These have been altered in the new design to produce a smoother variation in the on-axis longitudinal field. This is directly related to the magnitude of the non-linear transverse dependence of the fields [3], which has a strong effect on the emittance.

As in the SSRL design, the APS design incorporates a modification of the cathode end-wall to produce radially-focusing electric fields. In the present design, the structure producing these fields is further from the beam and less abrupt, giving a more linear dependence of $E_{r}$ on $r$. Evaluated at the cathode radius of $3 \mathrm{~mm}$, the peak nonlinear content of the radial and longitudinal fields have been reduced by a factor of three to four. 
2. Magnitude of $\mathrm{E}_{\mathrm{z}}$ at the cathode. Since both the SSRL and APS guns are designed for use with a magnetic compression system, the output longitudinal phase-space must exhibit a monotonic dependence of momentum on exit time. This reguires that particles arrive in the second cell of the gun behind the RF crest, which in turn requires either lowering the first-cell fields relative to the second $[1,3]$, or else moving the cathode end-wall back. In the SSRL gun, the peak on-axis fields in the two cells were in the ratio $\alpha=\mathrm{E}_{\mathrm{p} 2} / \mathrm{E}_{\mathrm{p} 1} \approx 3$. In the APS gun, the cathode end-wall was moved back $6 \mathrm{~mm}$, and the first-cell fields were increased to give $\alpha=1.63$. The ratio on the peak surface field to the cathode field has been increased from 0.13 to 0.28 . For the same peak surface field, the maximum beam momentum is about $75 \%$ higher. However, for the same maximum momemtum, the cavity wall power is approximately the same.

\section{GUN SIMULATIONS}

In this section, I report on the results of simulations of the gun using the fully electromagnetic PIC code spiffe [6]. The simulations were of the entire gun with a single bunch emitted from the cathode. Resistive effects in the cavity walls were not included, so the fields were created by ringing the individual cells, not by driving them to steady state.

Because the fields are time-varying, particles exiting the gun have a momenta from some maximum down to essentially zero; $45 \%$ to $55 \%$ of the charge is in the top $10 \%$ of the energy range. The transport line will incorporate a momentum filter to select just this "useful" portion of the beam.

Figure 3 shows the normalized RMS emittance of the useful beam versus the useful-beam charge, for a range of excitation levels and beam currents. The maximum current for each level corresponds to a current density of $\mathrm{J}=30 \mathrm{~A} / \mathrm{cm}^{2}$, which gives an approximate matched condition for the $\beta \approx 4$ cavity. As in the SSRL gun, the cathode has a $3 \mathrm{~mm}$ radius.

The emittances predicted are well below the desired level, particularly at low current. Note that the emittance of the useful beam decreases with current for $E_{\mathrm{p} 2}=80 \mathrm{MV} / \mathrm{m}$. This is a result of the selection of a fixed fractional momentum interval, which does not correspond to a fixed emission phase interval.

Additional simulations were done to assess the spacecharge-induced emittance growth for the gun beam in a drift following the gun. These indicate that significant emittance growth can occur, particularly at the higher current levels. For $E_{p 2}=60 \mathrm{MV} / \mathrm{m}$ and $\mathrm{J}=20 \mathrm{~A} / \mathrm{cm}^{2}$, the emittance increases $60 \%$ in a 50-cm drift. This effect motivates a short gun-to-linac transport system, which is in conflict with the needs of magnetic compression.

\section{TRANSPORT LINE}

As at SSRL, APS will employ an alpha-magnet-based magnetic bunching system $[7,3]$. The optimal system for low space-charge consists of an alpha-magnet and a total of $2 \mathrm{~m}$ of longitudinal drift space. A significantly shorter drift length would require a much stronger alpha-magnet, since the optimal gradient is inversely proportional to $\mathrm{L}^{2}$. Because of the magni- tude of space-charge effects on the drifting beam, however, a shortening of the transport line is under consideration, probably at the expense of increasing the bunch lengths from 1 to 2 ps to about 5 ps.

One significant problem at SSRL was emittance degradation due to quadrupole chromatic aberrations, particularly because a large momentum spread $( \pm 10 \%)$ was utilized. Simulations predicted emittance increases by factors of 5 to 10 , and these were born out by experiment. As implied above, the present project will use a smaller momentum spread ( $\pm 5 \%$ ). In addition, a strategy for correcting the chromatic aberrations was developed.

The common approach to correcting aberrations is to make a first-order design with the desired optical properties, usually building in symmetry to give cancellation of certain aberrations [8]. One then adds nonlinear elements to compensate as many of the remaining aberrations as possible. It was found that such a procedure was very difficult for the present case. In particular, a system with first-order optical symmetry tends to be longer, have greater phase advance, and have more elements than the solution used.

The solution adopted was to optimize the first- and higherorder properties of the transport line simultaneously. More precisely, the tracking program elegant [6] was used to optimize the heam transport by tracking the initial phase-space calculated with apiffe.

To understand this further, consider that the initial phasespace of the gun beam is roughly described by $x^{\prime}=\alpha x$, with $\langle x \delta\rangle=\left\langle x^{\prime} \delta\right\rangle=0$, where $\delta=\Delta p / p_{o}$. For simplicity, I assume that $\alpha$ is constant, giving an initially zero emittance beam.

If such a beam is sent through a thin-lens quadrupole of focal length $f_{0}$, then the slopes become $x_{1}^{\prime}=x^{\prime}-\frac{1-\delta}{f_{0}}$, and the geometric RMS ernittance becomes $\varepsilon_{1}=\pi \mathrm{x}_{\mathrm{ms}}^{2} \delta_{\mathrm{ms}} / \mathrm{f}_{\mathrm{o}}$. The immediate conclusion from this result is that one should avoid strong focusing and large beam sizes in designing the beamline. This is one reason that optically symmetric systems are more difficult, since oblaining a -I matrix from the start to midpoint requires strong quadrupoles or a relatively long system. One ends up seriously degrading the emittance simply by attempting to obtain the first-order optics solution.

Another lesson that can be drawn from this example is that the emittance degradation can be restored by another quadrupole. For example, if the initial beam has $\alpha=0$, then following the first quadrupole by another of equal but opposite strength at a distance of $2 f_{o}$ will result in an emittance proportional to $\delta_{\mathrm{ms}}^{2}-$-i.e., the degradation is essentially cancelled.

Unfortunately, this quadrupole pair does not form a useful optical element. The point of the example is to convince the reader that it is possible to recover the emittance even after degradation, and therefore that a beamline can be designed without separate chromatic correction elements. Figure 4 shows the RMS beam sizes and normalized emittances in the transport line designed for APS, using the optimization method just discussed. The initial phase space is from a spiffe simulation with $\mathrm{J}=5 \mathrm{~A} / \mathrm{cm}^{2}$ and $\mathrm{E}_{\mathrm{p} 2}=60 \mathrm{MV} / \mathrm{m}$. This gives just over $50 \mathrm{pC}$ 
of useful beam per pulse, which is adequate for our primary application. Future work will attempt to extend these results to transport with space charge.

\section{ACKNOWLEDGEMENTS}

The author wishes to acknowledge valuable consultations with Eiji Tanabe of AET, who built the gun, and with Jim Weaver of SSRL.

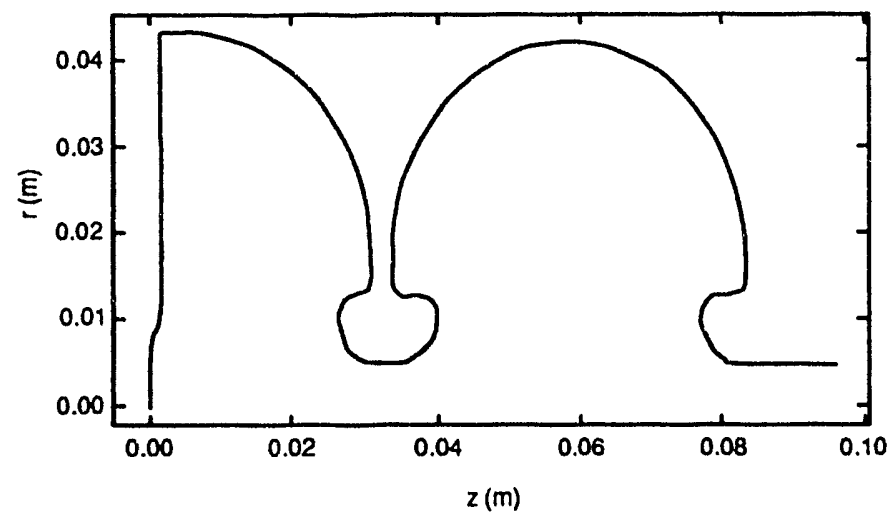

Figure 1

Cavity shape for the RF gun

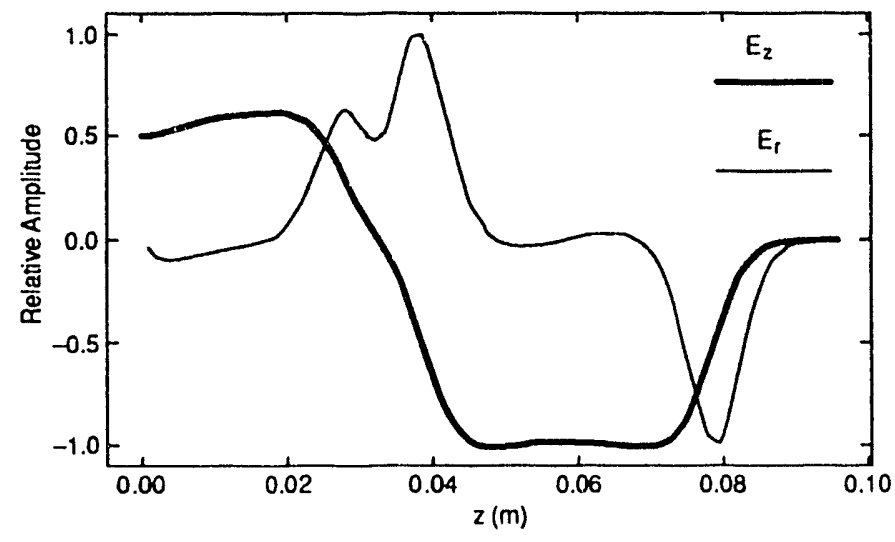

Figure 2

Electric field profiles for the gun cavity

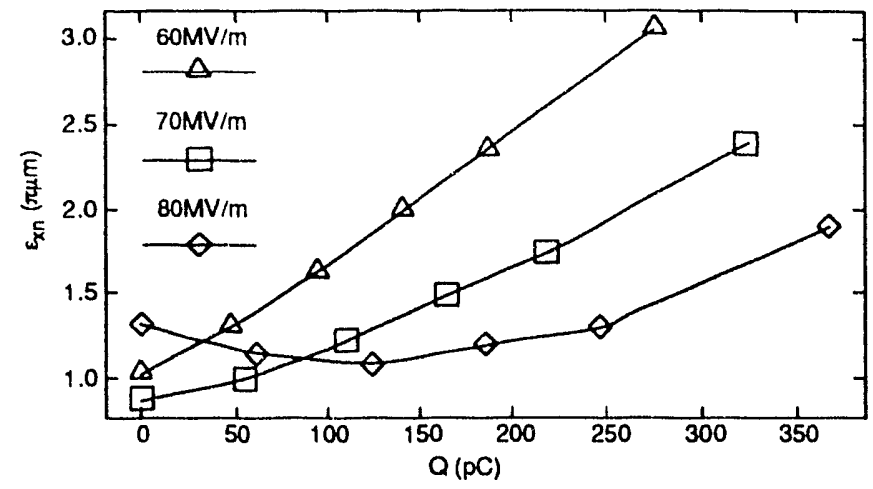

Figure 3

Normalized RMS emittance at the gun exit vs. useful charge per bunch

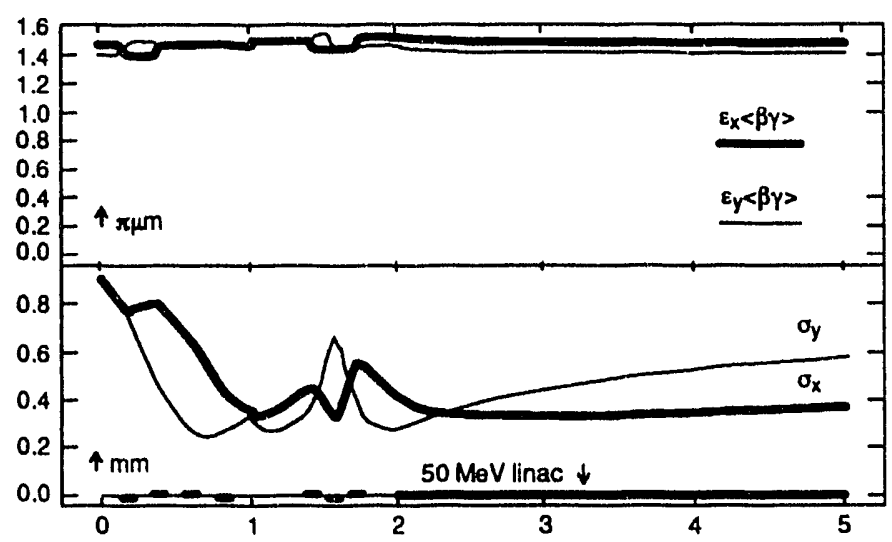

Figure 4

Geometric emittance along the transport line

\section{REFERENCES}

[1] M. Borland, et. al., "Performance of the $2 \mathrm{MeV}$ Microwave Gun for the SSRL150 MeV Linac," in Proceedings of the Linear Accelerator Conference, September 1990.

[2] J. W. Wang, et. al., "RF Breakdown Studies in Copper Electron Linac Structures," in Proceedings of the Particle Accelerator Conference, March 1989.

[3] M. Borland, "A High-Brightness Thermionic Microwave Electron Gun," SLAC-Report-402, 1991. Stanford University $\mathrm{Ph} . \mathrm{D}$. Thesis.

[4] A. Nassiri, et. al., "The Linac Injector for the ANL $7 \mathrm{GeV}$ Advanced Photon Source," in Proceedings of the Linear Accelerator Conference, September 1990.

[5] "Advanced Photon Source Conceptual Design Report," ANL-87-15, 1987.

[6] M. Borland. Unpublished program.

[7] H. A. Enge, "Achromatic Magnetic Mirror for Ion Beams," Review of Scientific Instruments, Vol. 34, No. 4, 1963.

[8] K. L. Brown, "A First- and Second-Order Matrix Theory for the Design of Beam Transport Systems and Charged Particle Spectrometers," SLAC-Report-75, 1982. 


\section{DISCLAIMER}

This report was prepared as an account of work sponsored by an agency of the United States Government. Neither the United States Government nor any agency thereof, nor any of their employees, makes any warranty, express or implied, or assumes any legal liability or responsibility for the accuracy, completeness, or usefulness of any information, apparatus, product, or process disclosed, or represents that its use would not infringe privately owned rights. Reference herein to any specific commercial product, process, or service by trade name, trademark, manufacturer, or otherwise does not necessarily constitute or imply its endorsement, recommendation, or favoring by the United States Government or any agency thereof. The views and opinions of authors expressed herein do not necessarily state or reflect those of the United States Government or any agency thereof. 

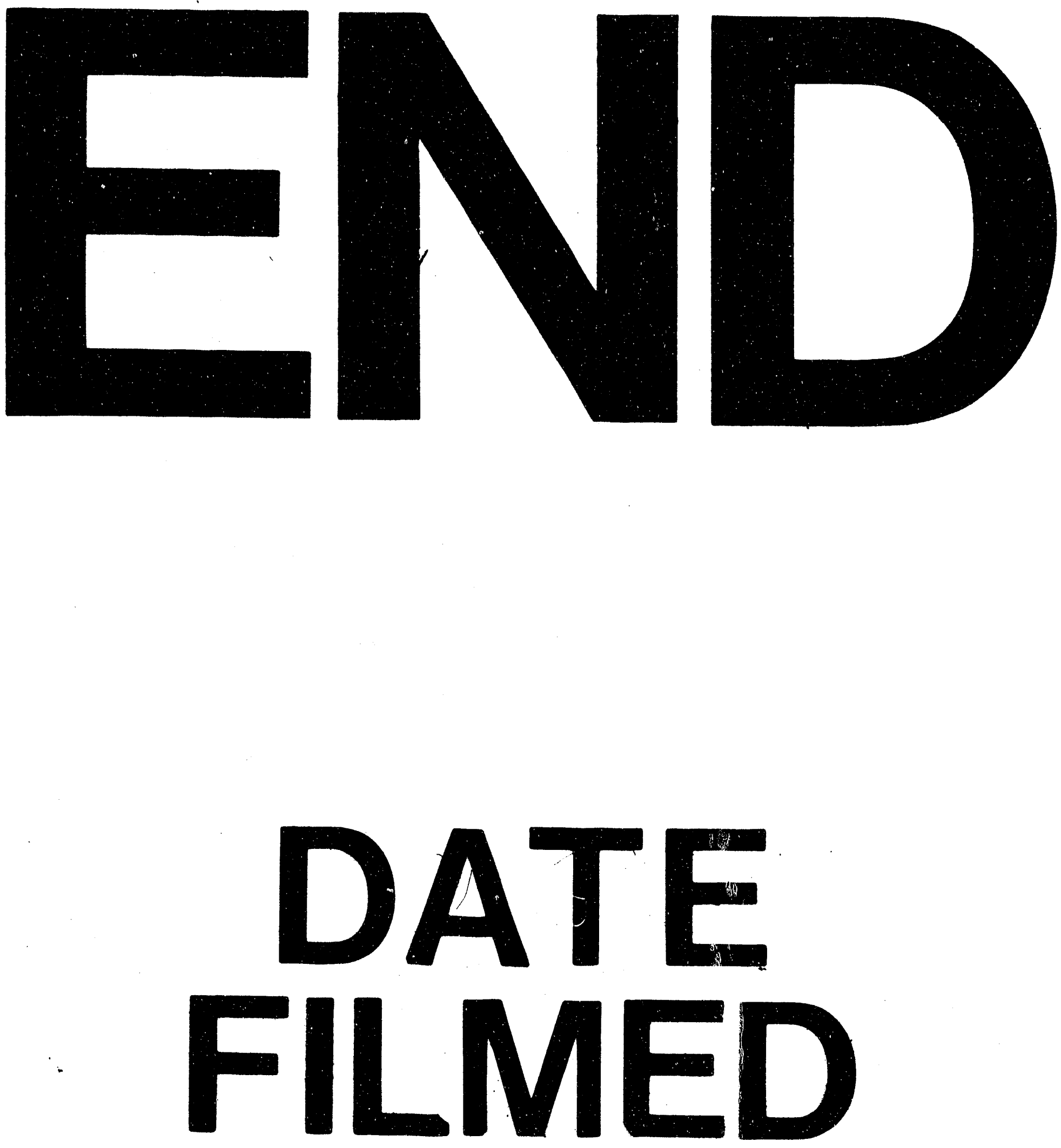

1

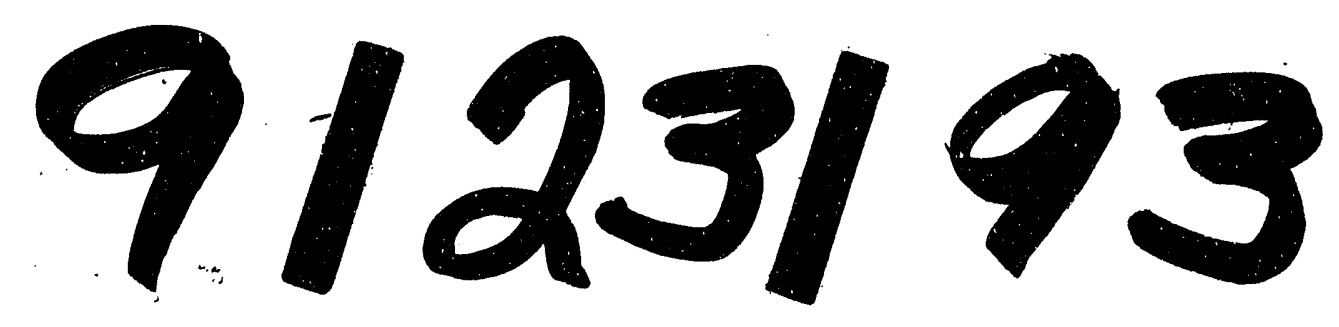


